\title{
Distributed full-consensus control of nonholonomic vehicles under non-differentiable measurement delays
}

\author{
Mohamed Maghenem Antonio Loría Emmanuel Nuño* Elena Panteley
}

\begin{abstract}
We address the problem of consensus control of second-order nonholonomic systems via distributed control under the assumption that each vehicle receives measured states from a set of neighbors, with a bounded, time-varying, but nondifferentiable delay. The controller that we propose guarantees full consensus, in the sense that a common consensus point may be reached both in the Cartesian positions on the plane and the orientations of all robots referred to a fixed frame. Our controller is smooth, hence time-varying. Notably, it relies on a property of persistency of excitation. Our main statement guarantees uniform global asymptotic stability. Also, we provide some simulation results to illustrate our theoretical findings.
\end{abstract}

Index Terms-Formation control, persistency of excitation, Lyapunov design, nonholonomic systems

\section{INTRODUCTION}

C ONSENSUS control of networked dynamical systems consists in steering certain variables of interest to a nonspecified common value. This problem is well studied and solved for a variety of dynamical systems, most often with linear dynamics, or nonlinear but with reliable interconnections; see for instance [1], [2] and references therein.

As pointed out in [2], consensus control of nonholonomic vehicles is particularly challenging since these systems are underactuated. But, also, because their nonholonomy prevents stabilization of a set-point via smooth time-invariant feedback [3], [4]. Indeed, consensus control is inherently a setpoint stabilization problem and it must be distinguished from formation-tracking control [5].

There are many decentralized controllers (either timevarying or non-smooth) for consensus of nonholonomic mobile robots proposed in the literature; see, for instance, [6]-[11] and the references therein, to mention a few. To distinguish these works, it is adequate to regard the type of consensus problem that is addressed. For instance, it may be partial consensus or full consensus. In the first case consensus in the Cartesian coordinates only is of interest while controlling the orientation

\footnotetext{
${ }^{*}$ Corresponding author E. Nuño is with the Dept. of Computer Science CUCEI, at University of Guadalajara, Guadalajara, Mexico. E-mail: emmanuel.nuno@cucei.udg.mx. M. Maghenem is with the University of California at Santa Cruz. E-mail: mmaghene@ucsc.edu. A. Loría and E. Panteley are with the CNRS. LSS-CentraleSupelec, 3 Rue Joliot Curie, 91192, France. E-mails: loria(panteley)@centralesupelec.fr. E. Panteley is also with ITMO University, Kronverkskiy av. 49, Saint Petersburg, 197101, Russia

This article has been supported by: the Government of Russian Federation (grant 08-08), by the French National Research Agency via the project HANDY (grant number ANR-18-CE40-0010), by ECOS-Nord contract no. M14M02, by the Mexican CONACyT Basic Scientific Research grant CB282807, and by CEFIPRA under the grant number 6001-A.
}

may be neglected [4] or it may consist in achieving a desired orientation [12]. In the case of full consensus the objective is for all robots to acquire common, non pre-specified position and orientation [6], [8].

Other distinctions pertain to the hypotheses made regarding the model, the formation, or the scenario. For instance, in [13] a consensus-based formation control problem is solved, albeit for specific parallel formations. In [8] and [9], the consensus problem is solved using smooth time-varying control laws that lead the agents to a given formation using only their orientation. In [7] consensus is solved for nonhoholonomic systems in chained form, under the assumption that the interconnections are affected by time delays.

In this paper we address the full-consensus problem. In contrast to [6] our controller is smooth time-varying and, different from [8], we establish uniform convergence of the error trajectories, which is not guaranteed by the analysis tools used in that reference. We also stress that our controllers rely on persistency of excitation, which has been widely used in the literature since its first use, for tracking control, in [14] and for set-point stabilization, in [15].

As in [7], we assume that the communications are affected by delays — see also [16] and [17]. However, in clear contrast with these references, we assume that delays are timevarying and non-differentiable. This is not an assumption of pure theoretical interest. It is motivated by, and apply to, scenarii in which a group of robots that are equipped with necessary sensors to measure their positions and orientations, transmit these data to their reachable neighbors over a wireless network. For instance, this may occur at the beginning of a rendez-vous-and-scout mission in which the robots are too far apart from each other, for relative-distance and line-ofsight sensors to be useful [18]. Naturally, the communication channels between any two pair of vehicles is bidirectional, so the graph topology is undirected, but the network introduces time-varying non-necessarily differentiable delays [19]. What enables to lift the unrealistic assumption on differentiability of the delays is that, in contrast to the literature (see e.g. [17]), our analysis does not involve Barbălat's lemma. Indeed, the utilization of this popular tool of analysis entails the recursive differentiation of the system's dynamics and, hence, of the time-delay function. Instead, we employ LyapunovKrasovskiî's method and output-injection arguments. To the best of our knowledge this has no precedent in the literature (other than [10]). 


\section{PROBLEM FORMULATION AND ITS SOLUTION}

Let us consider a swarm of $N$ autonomous, force-controlled, vehicles with dynamic model

$$
\begin{aligned}
\dot{x}_{i} & =v_{i} \cos \left(\theta_{i}\right) \\
\dot{y}_{i} & =v_{i} \sin \left(\theta_{i}\right) \\
\dot{\theta}_{i} & =\omega_{i}, \\
\dot{v}_{i} & =u_{v i} \\
\dot{\omega}_{i} & =u_{\omega i}, \quad i \leq N,
\end{aligned}
$$

where the variables $x_{i}$ and $y_{i}$ denote the Cartesian coordinates of the middle point on the vehicle's wheels axis relative to a fixed frame, $\theta_{i}$ denotes the vehicle's orientation with respect to the abcissae, and $v_{i}$ and $\omega_{i}$ denote the forward and angular velocities respectively. The model (1) corresponds to that of differential-wheel robots, but more general models, which include Lagrangian dynamics in place of Eqs. (1d)-(1e), are available, e.g., in [20]. Our main results apply to these models too since they are fully feedback-linearizable.

Due to evident physical constraints, consensus for noholonomic vehicles is formulated as the property that all the robots' Cartesian coordinates be equal modulo a certain offset with respect to an unknown barycenter with coordinates $z_{c}:=$ $\left[\begin{array}{ll}x_{c} & y_{c}\end{array}\right]^{\top}$, thereby constituting a formation. Thus, defining such an offset for each robot via the quantities $\left(\delta_{x i}, \delta_{y i}\right)$, we introduce the displaced-position variables

$$
z_{i}:=\left[\begin{array}{l}
x_{i}-\delta_{x_{i}} \\
y_{i}-\delta_{y_{i}}
\end{array}\right], \quad z=\operatorname{col}\left[z_{i}\right] \in \mathbb{R}^{2 N} .
$$

With this notation, the full consensus-formation may be characterized as the property that

$$
\begin{aligned}
& \lim _{t \rightarrow \infty} z_{i}(t)=z_{c}, \quad \lim _{t \rightarrow \infty} \theta_{i}(t)=\theta_{c} \\
& \lim _{t \rightarrow \infty} v_{i}(t)=0, \quad \lim _{t \rightarrow \infty} \omega_{i}(t)=0
\end{aligned}
$$

where both, the consensus position, $z_{c}$, and the orientation, $\theta_{c}$, are not imposed a priori, but depend on the network's topology and the systems' initial conditions. We use the qualifier "full" as opposed to "partial" in which case, orientation consensus is not pursued [12], or simply, orientation control is completely neglected [4], [18].

It might be argued that this problem may be solved by decoupling the consensus and the stabilization problems, i.e., by using an individual controller for each robot to steer it to a consensual reference trajectory $\xi_{i}(t)$ computed using a classical distributed consensus algorithm, such as

$$
\dot{\xi}_{i}=\sum_{j \in \mathcal{N}_{i}} a_{i j}\left(\xi_{j}-\xi_{i}\right), \quad \xi_{i}(0):=\left[z_{i}^{\top}(0) \theta_{i}(0)\right]^{\top}
$$

where $a_{i j} \geq 0 ; a_{i j}>0$ if the $i$ th and $j$ th vehicles communicate with each other and $a_{i j}=0$ otherwise. While such approach could be successfully used for Lagrangian systems (with holonomic constraints), it would appear ineffective for nonholonomic systems since such $\xi_{i}(t)$, in general, may be unfeasible - it may very well not satisfy equations (1a)-(1c). Thus, we define the errors

$$
e_{i}=\phi\left(\theta_{i}\right)^{\top} \sum_{j \in \mathcal{N}_{i}} a_{i j}\left(z_{j}-z_{i}\right),
$$

$$
s_{i}=\phi\left(\theta_{i}\right)^{\perp \top} \sum_{j \in \mathcal{N}_{i}} a_{i j}\left(z_{j}-z_{i}\right)
$$

where $\phi\left(\theta_{i}\right)^{\top}=\left[\cos \left(\theta_{i}\right) \sin \left(\theta_{i}\right)\right]$ and $\phi\left(\theta_{i}\right)^{\perp \top}=\left[\sin \left(\theta_{i}\right)-\right.$ $\left.\cos \left(\theta_{i}\right)\right]$. Note that $\operatorname{rank}\left[\phi\left(\theta_{i}\right) \phi\left(\theta_{i}\right)^{\perp}\right]=2$ for all $\theta_{i} \in \mathbb{R}$ therefore, $z_{i}=z_{c}$ for all $i \leq N$ if and only if $(s, e)=(0,0)$ [12]. That is, consensus is reached if and only if $(s, e, \theta)=$ $\left(0,0, \theta_{c}\right)$ and $\left(v_{i}, \omega_{i}\right) \rightarrow(0,0)$.

Next, in coherence with the setting described in the Introduction, we introduce the following hypothesis on the communications network.

Assumption 1 The interconnection graph is undirected, static and connected. Also, the communication during the transfer of information from the $j$ th to the $i$ th agent is affected by a time delay, determined by a function $t \mapsto T_{i j}(t)$, not necessarily continuous, but satisfying

$$
\underset{t \geq 0}{\operatorname{ess} \sup _{i j}}\left|T_{i j}(t)\right| \leq T^{*}
$$

where $T^{*}<\infty$ is known.

Now, beyond the mere convergence property expressed by the limits in (3), we solve the following open problem.

Full-Consensus with delays. Consider a network of $N$ nonholonomic robots, under Assumption 1. Then, design a dynamic controller such that all robots positions and orientation converge, globally, uniformly, and asymptotically, to a given formation pattern. More precisely, such that the origin for the closed-loop system,

$$
\left\{\left[e_{i} s_{i}\left(\theta_{i}-\theta_{c}\right) v_{i} \omega_{i}\right]^{\top}=0, \quad i \leq N\right\} \in \mathbb{R}^{5 N}
$$

is uniformly globally asymptotically stable.

The controller that solves this problem is defined as follows. For each robot $(i \leq N)$, the first control input is

$$
u_{v i}=-k_{d i} v_{i}+k_{p i} e_{d i}, \quad k_{d i}, k_{p i}>0,
$$

where $e_{d i}$ corresponds to the error (5) under the effect of measurement delay that is,

$$
e_{d i}=\phi\left(\theta_{i}\right)^{\top} \sum_{j \in \mathcal{N}_{i}} a_{i j}\left[z_{j}\left(t-T_{i j}\right)-z_{i}\right] .
$$

Correspondingly, based on (6) we introduce

$$
s_{d i}=\phi\left(\theta_{i}\right)^{\perp \top} \sum_{j \in \mathcal{N}_{i}} a_{i j}\left[z_{j}\left(t-T_{i j}\right)-z_{i}\right] .
$$

and the second control input, which is computed dynamically, i.e.,

$$
\begin{aligned}
& u_{\omega i}=-k_{\omega i} \omega_{i}-k_{\omega i} \dot{q}_{i}(t) \kappa_{i}\left(s_{d i}, e_{d i}\right) \\
&-\sum_{j \in \mathcal{N}_{i}} a_{i j}\left[\theta_{i}-\theta_{j}\left(t-T_{i j}(t)\right)\right]+k_{\alpha_{i}} \alpha_{i} \\
&-\sum_{j \in \mathcal{N}_{i}} a_{i j}\left[q_{i}(t) \kappa_{i}\left(s_{d i}, e_{d i}\right)\right. \\
&\left.-q_{j}\left(t-T_{i j}(t)\right) \kappa_{j}\left(s_{d d j}, e_{d d j}\right)\right] \\
& \dot{\alpha}_{i}=\quad-k_{\alpha i}^{\prime} \alpha_{i}-k_{I i} \omega_{i}+\dot{p}(t) \kappa_{i}\left(s_{d i}, e_{d i}\right) \\
& q_{i}^{(3)}+k_{\alpha i} \ddot{q}_{i}+k_{I i} \dot{q}_{i}=\dot{p},
\end{aligned}
$$


where $\alpha_{i}$ and $q_{i}$ are controller states; $k_{\alpha i}, k_{\alpha i}^{\prime}, k_{\omega i}$, and $k_{I i}>0$ are control gains; $q_{j}, \theta_{j}$ and $\kappa_{j}$ are received directly from the $j$ th neighbor - this is explained farther below, and $\kappa_{i}\left(s_{d i}, e_{d i}\right)$ is the $i$ th element of the vector

$$
\kappa\left(s_{d}, e_{d}\right):=\frac{1}{2}\left[\left(s_{d 1}^{2}+e_{d 1}^{2}\right) \cdots\left(s_{d N}^{2}+e_{d N}^{2}\right)\right]^{\top},
$$

where $e_{d}:=\operatorname{col}\left[e_{d i}\right]$ and $s_{d}:=\operatorname{col}\left[s_{d i}\right]$. The function $p$ : $\mathbb{R}_{\geq 0} \rightarrow \mathbb{R}$, whose derivative appears in (11b) and (11c), is smooth, bounded and has bounded derivatives, while $\kappa_{j}\left(s_{d d j}, e_{d d j}\right)$ is a quantity not measured, but received from the $j$ th agent, affected by a double delay. This is explained next.

The distributed dynamic controller (11) is implemented as follows. On one hand, each robot measures its own states, $\left(z_{i}, \theta_{i}, v_{i}, \omega_{i}\right)$, and it receives the corresponding positions and orientations from its $\mathcal{N}_{i}$ neighbors, albeit with a time-varying delay $T_{i j}(t)$, that is $z_{j}\left(t-T_{i j}(t)\right)$ and $\theta_{j}\left(t-T_{i j}(t)\right)$. Then, the $i$ th robot computes the errors using the fomulae (5) and (6), which, in view of the delays in the measurements, actually become $e_{d i}$ and $s_{d i}$, as in (9) and (10). With the latter, also the coefficients

$$
\kappa_{i}\left(s_{d i}, e_{d i}\right):=\frac{1}{2}\left[s_{d i}^{2}+e_{d i}^{2}\right], \quad i \leq N,
$$

are computed. Furthermore, the coefficients $q_{i}(t)$ and $\dot{q}_{i}(t)$ are generated using $(11 \mathrm{c})$.

On the other hand, according to the previous rationale, each neighbor robot computes and transmits its corresponding quantities $q_{j}(t)$ and $\kappa_{j}\left(s_{d j}, e_{d j}\right)$, computed by the $j$ th robot using the information from each of its own $\mathcal{N}_{j}$ neighbors. Thus, the data received by the $i$ th agent, with a delay $T_{i j}(t)$, correspond to $q_{j}\left(t-T_{i j}(t)\right)$ and

$$
\kappa_{j}\left(s_{d d j}, e_{d d j}\right):=\frac{1}{2}\left[s_{d d j}^{2}+e_{d d j}^{2}\right], \quad j \leq N,
$$

where $s_{d d j}$ and $e_{d d j}$ stand for $s_{d j}\left(t-T_{i j}(t)\right)$ and $e_{d j}\left(t-T_{i j}(t)\right)$ respectively. It is emphasized that these measurements are affected by a double delay and, mathematically, correspond to

$$
\begin{array}{r}
e_{d d j}=-\phi\left(\theta_{j}\left(t-T_{i j}(t)\right)\right)^{\top} \sum_{k \in \mathcal{N}_{j}} a_{j k}\left[z_{j}\left(t-T_{i j}(t)\right)\right. \\
\left.-z_{k}\left(t-T_{i j}(t)-T_{j k}\left(t-T_{i j}(t)\right)\right)\right] \\
s_{d d j}=-\phi\left(\theta_{j}\left(t-T_{i j}(t)\right)\right)^{\perp \top} \sum_{k \in \mathcal{N}_{j}} a_{j k}\left[z_{j}\left(t-T_{i j}(t)\right)\right. \\
\left.-z_{k}\left(t-T_{i j}(t)-T_{j k}\left(t-T_{i j}(t)\right)\right)\right] .
\end{array}
$$

The presence of this double delay considerably increases the difficulty to analyze the stability of the closed-loop system, but we stress that the expressions on the right-hand sides of (13) and (14) are not used in the implementation of the control law (11a); they are only provided for completeness.

\section{MAIN RESULT}

As is well-known, nonholonomic systems are not stabilizable to an equilibrium via smooth feedback, unless it is time-varying. We use a controller of the so-called $\delta$-persistently-exciting type [15], [12]. The stabilization mechanism of the control law (11a) relies on the excitation introduced by the function $\alpha_{i}$, which, roughly speaking, prevails as long as the consensus errors remain away from a $\delta$-neighborhood of the origin. Such excitation is provided by $\dot{p}_{i} \kappa_{i}\left(s_{d i}, e_{d i}\right)$ and, more particularly, by $\dot{p}_{i}$. Indeed, the term $\dot{p}_{i} \kappa_{i}\left(s_{d i}, e_{d i}\right)$ is $\delta$-persistently exciting and it may be regarded as an input in equation $(11 \mathrm{~b})$, which is reminiscent of a low-pass filter with output $\alpha_{i}$. Therefore, our main statement relies on the following hypothesis.

Assumption 2 The functions $p, \dot{p}, \ddot{p}$, and $p^{(3)}$ are bounded and, moreover, $\dot{p}(t)$ is persistently exciting, i.e., there exist $T_{p}$ and $\mu_{p}>0$ such that

$$
\int_{t}^{t+T_{p}} \dot{p}(s)^{2} d s \geq \mu_{p} \quad \forall t \geq 0 .
$$

Now, akin to adaptive control theory, a rule of thumb to define $\dot{p}_{i}$ is to make it periodic (although this is by no means necessary) and for it to have at least as many frequencies as robots in the swarm. Other related design parameters are the gains $k_{\alpha i}^{\prime}$ in (11b) and $k_{\alpha i}$ and $k_{I i}$ in (11c).

Theorem 1 Consider the systems (1) in closed loop with the distributed controllers (8)-(11). Let Assumption 1 hold and $k_{\omega m} \geq c_{1} / c_{2}$ where

$$
\begin{array}{r}
c_{1}=2 T^{* 2} N^{2} \bar{a}^{2}\left[\frac{1}{6}+2 c_{2}\right], \\
c_{2}=3 k_{\omega M}-2 \lambda_{N}(L)+\frac{3}{2} \frac{k_{I M}}{k_{\alpha m}},
\end{array}
$$

and $k_{m}$ and $k_{M}$ denote, respectively, the smallest and largest values of $k_{i}$, for all $i \leq N$ and $\bar{a} \geq a_{i j}$ for all $i, j \leq N$. Then, consensus is reached, both in Cartesian positions and orientation angles and the origin for the closed-loop system is uniformly globally asymptotically stable.

The proof of this statement relies on classical stability theory; specifically, on Lyapunov-Krasovskii theory for functional differential equations. First, we derive the closed-loop equations in a suitable form and then, we give the main guidelines to construct a Lyapunov-Krasovskii functional. The latter is done using the elegant Mazenc construction [21], [22].

\section{A. The closed-loop equations}

We start by introducing a multivariable model for the networked vehicles and a compact vector form for the controller (8)-(11).

Consider the equations (1) and let us define $\theta:=\operatorname{col}\left[\theta_{i}\right]$, $v:=\operatorname{col}\left[v_{i}\right], \omega=\operatorname{col}\left[\omega_{i}\right], \Phi(\theta)=\operatorname{blockdiag}\left[\phi\left(\theta_{i}\right)\right] \in \mathbb{R}^{2 N \times N}$, and the control inputs $u_{v}:=\operatorname{col}\left[u_{v i}\right]$ and $u_{\omega}:=\operatorname{col}\left[u_{\omega i}\right]$. The resulting multiagent system model is

$$
\begin{aligned}
\dot{z} & =\Phi(\theta) v \\
\dot{v} & =u_{v} \\
\dot{\theta} & =\omega \\
\dot{\omega} & =u_{\omega} .
\end{aligned}
$$


We use a Laplacian-based representation of the graph, defined by the matrix $L:=\left[\ell_{i j}\right] \in \mathbb{R}^{N \times N}$, where

$$
\ell_{i j}=\left\{\begin{array}{cc}
\sum_{j \in \mathcal{N}_{i}} a_{i j} & i=j \\
-a_{i j} & i \neq j
\end{array} .\right.
$$

Under Assumption 1, $L$ is symmetric, it has a single zeroeigenvalue and the rest of the spectrum is positive. Thus, $\operatorname{rank}(L)=N-1$.

For simple integrators, $\ddot{z}_{i}$, the first limit in (3a) is equivalent to having $\lim _{t \rightarrow \infty}\left[L \otimes I_{2}\right] z(t)=0$. In view of the nonholonomy, however, for the system (16a) the counterpart of the relation $\left[L \otimes I_{2}\right] z=0$ is that $e=0$ and $s=0$ simultaneously. To better see this, note that

$$
e=\Phi(\theta)^{\top} \mathcal{L} z, \quad s=\Phi(\theta)^{\perp \top} \mathcal{L} z,
$$

where $\mathcal{L}:=L \otimes I_{2}$ and the matrix $\left[\Phi(\theta) \Phi(\theta)^{\perp}\right]$ has full rank.

Next, with the purpose of obtaining a more compact layout of the controller equations, and only for the purpose of analysis, we introduce the change of coordinates,

$$
e_{\theta_{i}}:=\theta_{i}+q_{i}(t) \kappa_{i}\left(s_{i}, e_{i}\right)
$$

so that, substituting $\theta_{i}=e_{\theta_{i}}-q_{i}(t) \kappa_{i}\left(s_{i}, e_{i}\right)$ and

$$
\begin{aligned}
\theta_{j}\left(t-T_{i j}(t)\right)= & e_{\theta_{j}}\left(t-T_{i j}(t)\right) \\
& -q_{j}\left(t-T_{i j}(t)\right) \kappa_{j}\left(s_{d j}, e_{d j}\right)
\end{aligned}
$$

in the third term on the right-hand side of (11a), we obtain

$$
\begin{aligned}
u_{\omega i} & =-k_{\omega i} \omega_{i}-k_{\omega i} \dot{q}_{i}(t) \kappa_{i}\left(s_{d i}, e_{d i}\right) \\
& -\sum_{j \in \mathcal{N}_{i}} a_{i j}\left[e_{\theta_{i}}-e_{\theta_{j}}\left(t-T_{i j}(t)\right)\right]+k_{\alpha_{i}} \alpha_{i} \\
& +\sum_{j \in \mathcal{N}_{i}} a_{i j} q_{i}(t)\left[\kappa_{i}\left(s_{i}, e_{i}\right)-\kappa_{i}\left(s_{d i}, e_{d i}\right)\right] \\
& +\sum_{j \in \mathcal{N}_{i}} a_{i j} q_{j}\left(t-T_{i j}(t)\right)\left[\kappa_{j}\left(s_{d d j}, e_{d d j}\right)-\kappa_{j}\left(s_{d j}, e_{d j}\right)\right] .
\end{aligned}
$$

Then, let

$$
\begin{aligned}
\kappa_{d i} & :=\kappa_{i}\left(s_{d i}, e_{d i}\right)-\kappa_{i}\left(s_{i}, e_{i}\right), \\
\kappa_{d d j} & :=\kappa_{j}\left(s_{d d j}, e_{d d j}\right)-\kappa_{j}\left(s_{d j}, e_{d j}\right), \\
a_{i}\left(\dot{z}_{t}\right) & :=\sum_{j \in \mathcal{N}_{i}} a_{i j} \int_{t-T_{i j}(t)}^{t} \dot{z}_{j}(\sigma) d \sigma
\end{aligned}
$$

and

$$
6_{i}:=\sum_{j \in \mathcal{N}_{i}} a_{i j} q_{j}\left(t-T_{i j}(t)\right) \kappa_{d d j}
$$

and let us gather all terms in $N$-dimensional vectors and appropriate matrices of dimension $N \times N$. That is, we define the control gains $K_{\omega}:=\operatorname{diag}\left[k_{\omega_{i}}\right], K_{I}:=\operatorname{diag}\left[k_{I}\right]$, $K_{\alpha}:=\operatorname{diag}\left[k_{\alpha}\right], K_{\alpha}^{\prime}:=\operatorname{diag}\left[k_{\alpha}^{\prime}\right]$, the matrices $Q:=\operatorname{diag}\left[q_{i}\right]$, $\kappa_{d}:=\operatorname{diag}\left[\kappa_{d i}\right], D:=\operatorname{diag}\left[\sum_{j \in \mathcal{N}_{i}} a_{i j}\right]$, the vectors $\mathcal{A}\left(\dot{z}_{t}\right):=\operatorname{col}\left[\mathrm{a}_{i}\left(\dot{z}_{t}\right)\right]$ and $\mathcal{B}:=\operatorname{col}\left[\left[_{i}\right]\right.$, the error vectors with delays $e_{d}:=\operatorname{col}\left[e_{d i}\right]$ and $s_{d}:=\operatorname{col}\left[s_{d i}\right]$, and $e_{\theta}:=\operatorname{col}\left[e_{\theta i}\right]$. Thus, the distributed controller for the multiagent system (16) reads

$$
u_{\omega}=-K_{\omega}\left[\omega+\dot{Q}(t) \kappa\left(s_{d}, e_{d}\right)\right]-\left[L e_{\theta}+\mathcal{A}\left(\dot{e}_{\theta t}\right)\right]
$$

$$
\begin{aligned}
& +K_{\alpha} \alpha-D Q(t) \kappa_{d}\left(s, e, \theta, \dot{z}_{t}\right)+\mathcal{B}(t) \\
\dot{\alpha}=\quad & -K_{\alpha}^{\prime} \alpha-K_{I} \omega+\dot{p}(t) \kappa\left(s_{d}, e_{d}\right) .
\end{aligned}
$$

We stress, once more, that these expressions are used only for the purpose of analysis, but the controller is implemented using (8), (11) as explained in the previous section.

On the other hand, the distributed controller for the forward motion, $u_{v}$, is given by

$$
u_{v}=-K_{p} v-K_{d} e_{d}
$$

where $K_{p}:=\operatorname{diag}\left[k_{p_{i}}\right], K_{d}:=\operatorname{diag}\left[k_{d_{i}}\right]-c f$. Eq. (8).

Now, let us define

$$
e_{\omega}=\omega+\dot{Q}(t) \kappa(s, e), \quad e_{\alpha}=\alpha+Q(t) \kappa(s, e)
$$

and the closed-loop states

$$
X_{t}=\left[\begin{array}{lll}
v^{\top} & e^{\top} & s^{\top}
\end{array}\right]^{\top}, \quad X_{r}=\left[\begin{array}{lll}
e_{\theta}^{\top} & e_{\omega}^{\top} & e_{\alpha}^{\top}
\end{array}\right]^{\top} .
$$

We also introduce the matrices

$$
\begin{array}{rlrl}
\bar{e} & :=\operatorname{diag}\left[e_{i}\right], & \bar{s}:=\operatorname{col}\left[s_{i}\right], \\
\bar{e}_{\omega}:=\operatorname{diag}\left[e_{\omega_{i}}\right], & \bar{\kappa}:=\operatorname{diag}\left[\kappa_{i}\right] .
\end{array}
$$

With these notations, the closed-loop equations are obtained by replacing (24a) and (25) in (16d) and (16b), respectively, and using the expressions in (26). That is,

$$
\dot{X}_{t}=\left[\begin{array}{ccc}
-K_{d} & -K_{p} & 0 \\
0 & 0 & \dot{Q} \bar{\kappa}-\bar{e}_{\omega} \\
0 & -\dot{Q} \bar{\kappa}+\bar{e}_{\omega} & 0
\end{array}\right] X_{t}+\left[\begin{array}{c}
K_{p} \Phi^{\top} \mathcal{A}\left(\dot{z}_{t}\right) \\
\Phi^{\top} \mathcal{L} \Phi v \\
\Phi^{\perp \top} \mathcal{L} \Phi v
\end{array}\right]
$$

$\dot{X}_{r}=F_{r}\left(X_{r}, e_{\theta_{t}}\right)+\Psi\left(t, X_{t}, \theta, \dot{z}_{t}\right)$,

where

$$
\begin{aligned}
F_{r} & :=\left[\begin{array}{ccc}
0 & I_{N} & 0 \\
-L & -K_{\omega} & I_{N} \\
0 & -K_{I} & -K_{\alpha}
\end{array}\right] X_{r}-\left[\begin{array}{c}
0 \\
\mathcal{A}\left(\dot{e}_{\theta_{t}}\right) \\
0
\end{array}\right] \\
\Psi & :=\left[\begin{array}{c}
Q\left[\bar{e} \Phi^{\top}+\bar{s} \Phi^{\perp \top}\right] \mathcal{L} \Phi v \\
\dot{Q}\left[\bar{e} \Phi^{\top}+\bar{s} \Phi^{\perp \top}\right] \mathcal{L} \Phi v-\left[K_{\omega} \dot{Q}+\dot{Q}\right] \kappa_{d} \\
\ddot{Q}\left[\bar{e} \Phi^{\top}+\bar{s} \Phi^{\perp \top}\right] \mathcal{L} \Phi v-\dot{p} \kappa_{d}
\end{array}\right]-\left[\begin{array}{c}
0 \\
\mathcal{B}(t) \\
0
\end{array}\right]
\end{aligned}
$$

The first closed-loop equation, (28a), is the same as in [23], where only partial-consensus with measurement delays, but only in the Cartesian coordinates is addressed. Furthermore, in the absence of measurement delays, we recover the closedloop system [12, Eq. (21)]. Therefore, we can rely on the analyses in the previous references to make the following reasoning. Firstly, after [23], it may be established that $X_{t} \rightarrow 0$ provided that $K_{p}$ and $K_{d}>0$ and $\dot{Q}$ is persistently exciting, that is, if there exist $\mu$ and $T>0$ such that

$$
\int_{t}^{t+T} \dot{Q}(s) d s \geq \mu I \quad \forall t \geq 0 .
$$

This is the case under Assumption 2.

Furthermore, this holds uniformly in the error trajectories $e_{\omega}(t)$ hence, the "translational motion" error dynamics (28a) may be regarded as a system that is decoupled from the "angular motion" error dynamics, (28b). In turn, in the latter equation, $\Psi$ is reminiscent of a (vanishing) perturbation. It may be reasonably assumed that a cascades argument may be invoked to conclude the convergence of all state trajectories to the origin. 


\section{B. Stability analysis}

The previous rationale is at the basis of the following statement, which covers the main results in [12] and [23] and establishes uniform global asymptotic stability of the origin for (28) provided that $\mathcal{B} \equiv 0$. Farther below, Lemma 2 establishes that the overall system is robust with respect to the perturbation $\mathcal{B}(t)$ and uniform asymptotic stability is preserved.

Lemma 1 Consider the system (28) with $\mathcal{B} \equiv 0$, under Assumptions 1 and 2 and the condition (15). Then, the set

$$
\mathcal{D}:=\left\{\left(X_{t}, X_{r}\right) \in \mathbb{R}^{6 N}:\left(X_{t}, L e_{\theta}, e_{\omega}, e_{\alpha}\right)=0\right\}
$$

is uniformly globally asymptotically stable.

Sketch of Proof. First, from [23], we recall the functional

$$
V_{1}=v^{\top} K_{p}^{-1} v+z^{\top} \mathcal{L} z+\int_{-T^{*}}^{0} \int_{t+\theta}^{t}|\dot{z}(\tau)|^{2} d \tau d \theta,
$$

which is positive definite and radially unbounded with respect to $X_{t}$-because $\lambda_{2}(L) z^{\top} \mathcal{L} z \leq|e|^{2}+|s|^{2} \leq \lambda_{N}(L) z^{\top} \mathcal{L} z$ where $\lambda_{i}$ is the $i$ th eigenvalue - and its total along the trajectories of (28a) satisfies

$$
\begin{aligned}
\dot{V}_{1} & \leq-v^{\top} K_{p}^{-1} K_{d} v-Y\left(\dot{z}_{t}\right) \\
Y\left(\dot{z}_{t}\right) & :=\frac{1}{2 \bar{a}^{2} N T^{*}} \sum_{j=1}^{N} \sum_{i=1}^{N} a_{i j}^{2}\left|\int_{t-T_{i j}}^{t} \dot{z}_{i}(\tau) d \tau\right|^{2} .
\end{aligned}
$$

Hence, $\dot{V}_{1}$ is negative semidefinite and it may be established, using standard arguments based on Barbalăt's lemma, that $Y\left(\dot{z}_{t}\right)$ and $v \rightarrow 0$.

Next, for the angular-motion dynamics, (28b), we introduce the functional

$$
\begin{gathered}
V_{2}:=c_{2} V_{21}+e_{\theta}^{\top} L e_{\omega}+c_{1} \int_{-T^{*}}^{0} \int_{t+h}^{t}\left|\dot{e}_{\theta}(s)\right|^{2} d s d h \\
V_{21}:=\left[e_{\theta}^{\top} L e_{\theta}+\left|e_{\omega}\right|^{2}+e_{\alpha}^{\top} K_{I}^{-1} e_{\alpha}\right]
\end{gathered}
$$

where $c_{2}$ and $c_{1}$ are positive constants defined in (15). $V_{2}$ is positive definite, radially unbounded and decrescent; it satisfies

$$
\frac{c_{2}}{2} V_{21} \leq V_{2} \leq 2 c_{2} V_{21}+2 c_{1} T^{*} \int_{t-T^{*}}^{t}\left|\dot{e}_{\theta}(s)\right|^{2} d s
$$

so $V_{2}$ qualifies as a Lyapunov-Krasovskii functional candidate. Then, we use the fact that $K_{\omega}>I_{N}$ (see Theorem 1), and (15) to find, after some long, but straightforward computations, that

$$
\dot{V}_{2}=-\frac{1}{2} c_{2}\left[e_{\alpha}^{T} K_{I}^{-1} K_{\alpha} e_{\alpha}+e_{\omega}^{T} K_{\omega} e_{\omega}\right]-\frac{1}{2} e_{\theta}^{T} L^{2} e_{\theta} .
$$

Finally, we introduce the following Lyapunov-Krasovskii functional,

$$
\begin{aligned}
\mathcal{V}\left(t, X_{t}, X_{r}, \dot{z}_{t}, \dot{e}_{\theta t}\right)= & W\left(t, X_{t}, V_{1}, \dot{z}_{t}\right) \\
& +\rho_{1}\left(V_{1}\right) V_{2}\left(X_{r}, \dot{e}_{\theta t}\right)+\rho_{2}\left(V_{1}\right) V_{1} .
\end{aligned}
$$

The functional $W$ is given by the following expression,

$$
\begin{aligned}
W= & \gamma\left(V_{1}\right) V_{1}+V_{1} \kappa(s, e)^{\top}\left[\int_{t}^{t+T} \dot{Q}(s) d s\right] \kappa(s, e) \\
& +\alpha\left(V_{1}\right) e^{\top} v-\varepsilon V_{1} e^{\top} \dot{Q}(t) s+\varepsilon b_{q} \lambda_{N}(L) V_{1}^{2}
\end{aligned}
$$

$$
+\left[\lambda_{N}(L)+\left|K_{p}\right|\right] \alpha\left(V_{1}\right) V_{1}
$$

-cf. [12]. The functions $\rho_{1}, \rho_{2}, \alpha$, and $\gamma$, as well are polynomials of $V_{1}$ with positive coefficients. These and the constant $\varepsilon>0$ are chosen so that $\mathcal{V}$ be positive definite and radially unbounded, and its derivative be negative definite. This is possible provided that $k_{\omega m} \geq c_{1} / c_{2}$, as required in Theorem 1 , and (15) hold — see [10] for detailed lengthy computations. As a matter of fact, these can be chosen so that

$$
\begin{aligned}
\dot{\mathcal{V}} \leq & -\frac{\mu}{4 T} V^{3}-\frac{\rho_{1}(V)}{8}\left[c_{2} e_{\omega}^{\top} K_{d \theta} e_{\omega}+e_{\theta}^{\top} K_{p \theta} e_{\theta}\right] \\
& -\frac{1}{4} \gamma(V) v^{\top} K_{d t} K_{p t}^{-1} v-\frac{1}{8} \alpha(V) e^{\top} K_{p t} e
\end{aligned}
$$

so the statement follows.

Lemma 2 Under the conditions of Theorem 1 the set (29) for the closed-loop system (28) is uniformly globally asymptotically stable.

Sketch of Proof. The proof relies on a standard outputinjection argument, akin to [24, Proposition ] and the fact that $\mathcal{B}(t)$ is uniformly bounded and square integrable. Firstly, we observe that the solutions $X_{t}(t)$ of (28a) are uniformly globally bounded; this follows by integrating on both sides of (31). In addition, the nominal system $\dot{X}_{r}=F_{r}\left(X_{r}, e_{\theta_{t}}\right)$ in (28b) is input-to-state stable and the "input" $\Psi$ is bounded, so the solutions of (28b), $X_{r}(t)$, are also uniformly bounded. Secondly, some computations establish that there exist a constant $c$, independent of the initial times, such that

$$
|\mathcal{B}(t)|^{2} \leq c\left|\sum_{k \in \mathcal{N}_{j}} a_{k j}^{2} \int_{t-T_{k j}-T^{*}}^{t-T^{*}} \dot{z}_{j}(\delta)\right|^{2},
$$

so, in view of (31), it follows that $\mathcal{B}(t)$ is square integrable. The result follows from this, uniform global boundedness of the solutions, and uniform global asymptotic stability of $\mathcal{D}$ for the system with $\mathcal{B} \equiv 0$ (see Lemma 1 ).

\section{A Numerical EXAMPLE}

In this section we illustrate our main result via a numerical example consisting in performing full-consensus-based formation control of four identical unicycle-type robots. They are required to meet at an unknown rendez-vous point, forming a rhomboid. To that end we set the offsets $\left(\delta_{x i}, \delta_{y i}\right)$, for each $i \leq 4$, to $(0,2),(-2,0),(0,-2)$, and $(2,0)$. The initial positions, in the format $\left(x_{i}(0), y_{i}(0)\right)$, are set to $(5,-4),(7,3)$, $(-2,-5)$, and $(-4,5)$ while all the robots initially point East, i.e., $\theta_{i}(0)=0$ for all $i \leq 4$.

The control gains are set to $K_{p}=60, K_{d}=40$, $K_{\alpha}=K_{\alpha}^{\prime}=40, K_{I}=8$, and $K_{w}=40$. The persistently exciting function, $\dot{p}(t)$, is defined a periodic function of five frequencies,

$$
\dot{p}(t)=\pi[2 \sin (0.5 t)+\sin (2 t)+3 \sin (3 t)+\sin (t)-\sin (5 t)] .
$$

For simplicity, the time-varying delay is taken equal for all the interconnections. It is generated by a normal Gaussian distribution with a mean of 0.3 and a variance of 0.0003 , which generates a function bounded in norm by $T^{*}=0.37$. 


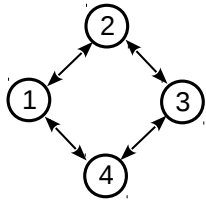

$$
L=\left[\begin{array}{rrrr}
2 & -1 & 0 & -1 \\
-1 & 2 & -1 & 0 \\
0 & -1 & 2 & -1 \\
-1 & 0 & -1 & 2
\end{array}\right]
$$

Fig. 1. Interconnection Graph and Laplacian matrix

The robots are interconnected in a closed undirected cycle as illustrated in Fig. 1.

Fig. 2 illustrates the paths described by the robots on the plane. The initial positions are marked by '*' and the final orientations are represented by arrows. It can be observed that the robots reach the desired formation with an a priori unknown center at $z_{c} \approx(1.27,-1.61)$ and a common orientation angle of $-177.66 \mathrm{deg}$.

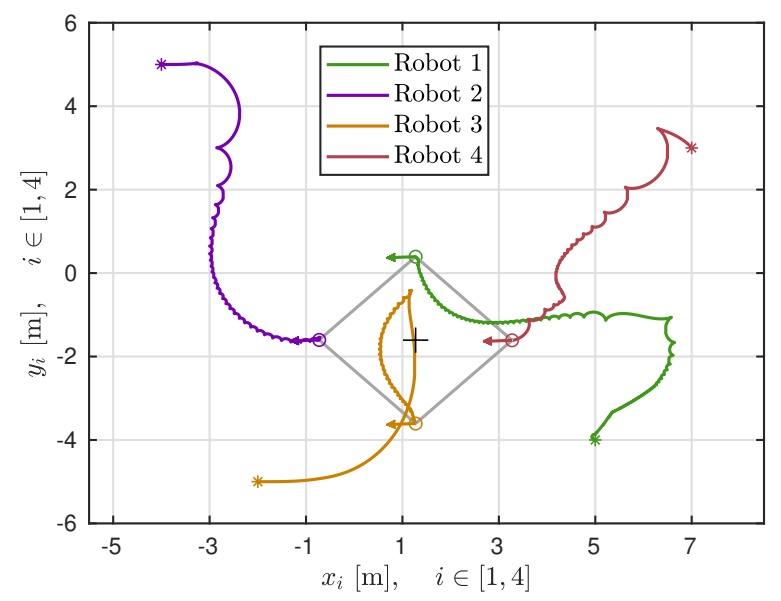

Fig. 2. Trajectories and formation of the network of mobile robots.

\section{CONCLUSiOnS}

We presented a decentralized controller for the the fullconsensus problem of multiple second-order nonholonomic vehicles with time-varying delayed communications. That is, our controller guarantees that all vehicles' orientations and Cartesian positions converge to a common equilibrium point. For the Cartesian positions an offset for each vehicle is specified hence, the vehicles may acquire any formation. Only few other results exist in the literature guaranteeing full consensus and with time-varying delays, but none in which delays may be non-differentiable, let alone for second-order systems.

The controller relies on persistency of excitation to overcome the difficulties imposed by the nonholonomy. Although such controllers guarantee strong properties such as uniform convergence of the error trajectories, they are difficult to tune and may entail oscillatory motions. Moreover, our controller relies on state feedback. Research on how to improve performance as well as on output-feedback control designs is to be carried out.

\section{REFERENCES}

[1] W. Ren and R. W. Beard, Distributed consensus in multivehicle cooperative control. Springer verlag, 2005.
[2] Y. Cao, W. Yu, W. Ren, and G. Chen, "An overview of recent progress in the study of distributed multi-agent coordination," IEEE Trans. on Industrial Informatics, vol. 9, no. 1, pp. 427-438, 2013.

[3] R. W. Brockett, "Asymptotic stability and feedback stabilization," Differential geometric control theory, vol. 27, no. 1, pp. 181-191, 1983.

[4] Z. Lin, B. Francis, and M. Maggiore, "Necessary and sufficient graphical conditions for formation control of unicycles," Automatic Control, IEEE Transactions on, vol. 50, no. 1, pp. 121-127, 2005.

[5] K.-K. Oh, M.-C. Park, and H.-S. Ahn, "A survey of multi-agent formation control," Automatica, vol. 53, pp. 424-440, 2015.

[6] D. Dimarogonas and K. Kyriakopoulos, "On the rendezvous problem for multiple nonholonomic agents," Automatic Control, IEEE Transactions on, vol. 52, no. 5, pp. 916-922, 2007.

[7] W. Dong and J. Farrell, "Consensus of multiple nonholonomic systems," in Decision and Control, 2008. CDC 2008. 47th IEEE Conference on, pp. 2270-2275, IEEE, 2008.

[8] C. Yang, W. Xie, C. Lei, and B. Ma, "Smooth time-varying formation control of multiple nonholonomic agents," in Proceedings of the 2015 Chinese Intelligent Systems Conference, pp. 283-291, Springer, 2016.

[9] Z. Peng, G. Wen, A. Rahmani, and Y. Yu, "Distributed consensusbased formation control for multiple nonholonomic mobile robots with a specified reference trajectory," International Journal of Systems Science, vol. 46, no. 8, pp. 1447-1457, 2015.

[10] M. Maghenem, Stability and stabilization of networked varying systems. $\mathrm{PhD}$ thesis, Univ Paris Saclay, Gif sur Yvette, 2017.

[11] E. Kranakis, D. Krizanc, and S. Rajsbaum, "Mobile agent rendezvous: A survey," in Structural Information and Communication Complexity, pp. 1-9, Springer, 2006.

[12] M. Maghenem, A. Bautista-Castillo, E. Nuño, A. Loría, and E. Panteley, "Consensus of multi-agent systems with nonholonomic restrictions via Lyapunov's direct method," IEEE Control Systems Letters, vol. 3, no. 2, pp. 344-349, 2019.

[13] X. He and Z. Geng, "Consensus-based formation control for nonholonomic vehicles with parallel desired formations," International Journal of Control, vol. 0, no. 0, pp. 1-14, 2019.

[14] E. Panteley, E. Lefeber, A. Loría, and H. Nijmeijer, "Exponential tracking of a mobile car using a cascaded approach," in IFAC Workshop on Motion Control, (Grenoble, France), pp. 221-226, 1998.

[15] A. Loría, E. Panteley, and A. Teel, "A new persistency-of-excitation condition for UGAS of NLTV systems: Application to stabilization of nonholonomic systems," in Proc. 5th. European Contr. Conf., (Karlsrühe, Germany), pp. 1363-1368, 1999.

[16] H. Wang, "Consensus of networked mechanical systems with communication delays: A unified framework," IEEE Transactions on Automatic Control, vol. 59, no. 6, pp. 1571-1576, 2014.

[17] E. Nuño, T. Hernández, M. Maghenem, A. Loría, and E. Panteley, "Leaderless consensus-based formation control of multiple nonholonomic mobile robots with interconnecting delays," in Proc. IEEE American Control Conference, (Philadelphia, PA, USA), pp. 4659-4664, 2019. DOI: 10.23919/ACC.2019.8814391.

[18] A. Roza, M. Maggiore, and L. Scardovi, "A smooth distributed feedback for global rendezvous of unicycles," IEEE Transactions on Control of Network Systems, vol. 5, pp. 640-652, March 2018.

[19] A. Abdessameud, I. Polushin, and A. Tayebi, "Synchronization of Lagrangian systems with irregular communication delays," IEEE Transactions on Automatic Control, vol. 59, no. 1, pp. 187-193, 2014.

[20] K. D. Do, Z.-P. Jiang, and J. Pan, "A global output-feedback controller for simultaneous tracking and stabilization of unicycle-type mobile robots," IEEE Trans. on Robotics Automat., vol. 20, no. 3, pp. 589594, 2004.

[21] F. Mazenc, "Strict Lyapunov functions for time-varying systems," $A u$ tomatica, vol. 39, no. 2, pp. 349-353, 2003.

[22] M. Malisoff and F. Mazenc, Constructions of Strict Lyapunov functions. London: Springer Verlag, 2009.

[23] M. Maghenem, E. Nuño, A. Loría, and E. Panteley, "Decentralized partial-consensus control of nonholonomic vehicles over networks with interconnection delays," in To appear in the Proc. IEEE American Control Conference (ACC), (Denver, CO, USA), p. 2020.

[24] E. Panteley, A. Loría, and A. Teel, "Relaxed persistency of excitation for uniform asymptotic stability," IEEE Trans. on Automat. Contr., vol. 46, no. 12 , pp. $1874-1886,2001$. 